\title{
On the Enumeration of One-Factorizations of Complete Graphs Containing Prescribed Automorphism Groups
}

\author{
By E. Seah and D. R. Stinson
}

\begin{abstract}
In this paper we use orderly algorithms to enumerate (perfect) one-factorizations of complete graphs, the automorphism groups of which contain certain prescribed subgroups. We showed that, for the complete graph $K_{12}$, excluding those one-factorizations containing exactly one automorphism of six disjoint cycles of length two, there are precisely 56391 nonisomorphic one-factorizations of $K_{12}$ with nontrivial automorphism groups. We also determined that there are precisely 21 perfect one-factorizations of $K_{14}$ that have nontrivial automorphism groups.
\end{abstract}

1. Introduction. A one-factorization (OF) of a complete graph $K_{2 n}$ is a partition of the edge-set of $K_{2 n}$ into $2 n-1$ one-factors, each of which contains $n$ edges that partition the vertex-set of $K_{2 n}$.

Although it is well known that there exists an OF of $K_{2 n}$ for every positive integer $n$ [5], the problem of determining $N(2 n)$, the number of pairwise nonisomorphic OFs of $K_{2 n}$, appears to be a difficult one. Wallis [16] gave a lower bound for $N(2 n)$ and showed that $N(2 n) \geq 2$ for $n \geq 4$. Later, Lindner et al. (see [9]) and Cameron (see [1] and [2]) proved that $N(2 n)$ goes to infinity with $n$. The best known lower bound on $N(2 n)$ is derived by Cameron in [2]. He proved the following result.

THEOREM 1.1. For sufficiently large nonnegative $n$, there holds $\ln N(2 n) \approx$ $2 n^{2} \ln 2 n$.

In fact, the exact values of $N(2 n)$ are only known for a few small values of $n$, as stated in the following theorem.

THEOREM 1.2. For $2 n=2,4$, and 6 , there is a unique one-factorization of $K_{2 n}$ (up to isomorphism). There are precisely 6 nonisomorphic one-factorizations of $K_{8}$ and 396 nonisomorphic one-factorizations of $K_{10}$.

$N(8)=10$ is proved in [3] and [15] and $N(10)=396$ is shown in [4].

A perfect $\mathrm{OF}$ is an OF in which every pair of distinct one-factors forms a Hamiltonian cycle of the graph. Denote $N_{p}(2 n)$ to be the number of nonisomorphic perfect OF of $K_{2 n}$. As in the case of $N(2 n)$, the exact values of $N_{p}(2 n)$ are only known for a few small values of $n$ :

THEOREM 1.3. For $2 n=2,4,6,8$, and 10 , there is a unique perfect onefactorization of $K_{2 n}$ (up to isomorphism). There are precisely 5 nonisomorphic perfect one-factorizations of $K_{12}$ [10].

Received February 17, 1987.

1980 Mathematics Subject Classification (1985 Revision). Primary 05C30, 05C45, $20 \mathrm{~B} 25$. 
In [13], we enumerated nonisomorphic OFs and Howell designs for the graph $K_{10}$ minus a one-factor. In [14], we constructed some perfect OFs of $K_{14}$. The methods used in the two papers are very similar and are known as orderly algorithms. In using these algorithms, we construct only nonisomorphic OFs, by eliminating isomorphic structures as they are being constructed. The same algorithms could be used to enumerate the OFs of $K_{12}$. However, the number of intermediate (nonisomorphic) structures grows at such an astronomical rate that a complete enumeration of $N(12)$ (or $N_{p}(14)$ ) is not feasible at this point in time. Consequently, we modified the orderly algorithms in [13] and [14] to restrict our enumeration to OFs of $K_{12}$, and perfect OFs of $K_{14}$, that contain certain subgroups in their automorphism groups (these algorithms are described in the remainder of this paper). We proved the following results.

THEOREM 1.4. There are precisely 56391 nonisomorphic one-factorizations of $K_{12}$ that have automorphism groups which are nontrivial and do not consist of 6 disjoint cycles of size 2 .

THEOREM 1.5. There are precisely 21 nonisomorphic perfect one-factorizations of $K_{14}$ having nontrivial automorphism groups.

For those readers who are interested in orderly algorithms, we recommend the paper by Read [12].

2. Definitions and Preliminaries. To explain the orderly algorithms used in this paper, we need the following definitions.

We first need to define orderings on edges, one-factors, etc, of $K_{2 n}$. All orderings are defined lexicographically.

Suppose the vertices are numbered $1, \ldots, 2 n$. We define an edge to be an ordered pair $\left(p, p^{\prime}\right)$ with $1 \leq p<p^{\prime} \leq 2 n$. Then given two edges $e_{1}=\left(p_{1}, p_{1}^{\prime}\right)$ and $e_{2}=\left(p_{2}, p_{2}^{\prime}\right)$, we say $e_{1}<e_{2}$ if either of the following is true: (1) $p_{1}<p_{2}$, (2) $p_{1}=p_{2}$ and $p_{1}^{\prime}<p_{2}^{\prime}$. We define a one-factor $f$ as an ordered set of edges, i.e., $f=\left(e_{1}, e_{2}, \ldots, e_{n}\right)$, where $e_{i}<e_{j}$ whenever $i<j$.

Let $A$ be any subgroup of $\mathbf{S}_{2 n}$, the symmetric group of $2 n$ elements. The onefactors of $K_{2 n}$ must form disjoint orbits under the action of the group $A$. We are only interested in those orbits which contain edge-disjoint one-factors. We say these are the eligible orbits under the action of $A$.

We order the one-factors in an orbit $O=\left(f_{1}, f_{2}, f_{3}, \ldots, f_{k}\right)$ such that $f_{i}<f_{j}$ whenever $i<j$. We say that $f_{1}$ is the representative of the orbit and write $f_{1}=$ $\operatorname{rep}(O)$. We define $L(O)=k$ to be the length of the orbit $O$.

We are now ready to define orderings on orbits and OFs. For two orbits $O_{1}$ and $O_{2}$, we say $O_{1}<O_{2}$ if $\operatorname{rep}\left(O_{1}\right)<\operatorname{rep}\left(O_{2}\right)$. An OF $F$ is then written as a list of orbits $\left(O_{1}, O_{2}, \ldots, O_{m}\right)$, where $O_{i}<O_{j}$ whenever $i<j$. Note that $\sum_{1 \leq i \leq m} L\left(O_{i}\right)=2 n-1$.

A partial OF $F_{i}=\left(O_{1}, O_{2}, \ldots, O_{i}\right)$ is written as a list of $i$ orbits. We also define $R=\sum_{1 \leq j \leq i} L\left(O_{j}\right)$ to be the rank of $F_{i}$. Note that when $R=2 n-1$, we have a (complete) OF.

Let $\mathrm{U}_{i}$ be the set of all one-factors containing the edge $(1, i+1)$. We say that a partial OF $F_{i}=\left(O_{1}, O_{2}, \ldots, O_{i}\right)$ is proper if it contains one one-factor from each 
of $\mathrm{U}_{1}, \ldots, \mathbf{U}_{k}$ where $\operatorname{rep}\left(O_{i}\right)$ contains the edge $(1, k+1)$. It is not difficult to see that if $F_{i}=\left(O_{1}, O_{2}, \ldots, O_{i}\right)$ is proper, and $1 \leq j \leq i$, then $F_{j}\left(O_{1}, O_{2}, \ldots, O_{j}\right)$ is also proper. Also, note that any (complete) OF is proper.

For two proper partial OFs $F_{i}=\left(O_{1}, O_{2}, \ldots, O_{i}\right)$ and $G_{i}=\left(P_{1}, P_{2}, \ldots, P_{i}\right)$ that have the same number of orbits $i$, we say that $F_{i}<G_{i}$ if there exists an integer $k$ $(1 \leq k \leq i)$ such that $\operatorname{rep}\left(O_{l}\right)=\operatorname{rep}\left(P_{l}\right)$ for all $l<k$ and $\operatorname{rep}\left(O_{k}\right)<\operatorname{rep}\left(P_{k}\right)$.

A proper partial OF $F_{i}$ is said to be $A$-canonical if $F_{i}^{\alpha} \geq F_{i}$ for all $\alpha \in M\left(F_{i}\right)$, where $M\left(F_{i}\right)=\left\{\alpha: \alpha \in \mathbf{S}_{2 n}\right.$, and $\alpha$ maps any orbit of $F_{i}$ into an orbit of the same length $\}$. A one-factorization $F_{i}$ that is $A$-canonical is in general not canonical as defined in [13] and [14], since the eligible orbits (one-factors) depend on the prescribed group $A$. (Some one-factors may not belong to any eligible orbits.) Given an $A$-canonical OF $F$, we can determine its canonical form by, for example, mapping all the one-factors of $F$ into the smallest one-factor of $K_{2 n}$, $((1,2),(3,4), \ldots,(2 n-1,2 n))$. The smallest OF resulting from these mappings is the canonical representation of $F$ (see [13]).

It is easy to see that if two proper partial OFs of rank $R$ (not necessarily having the same number of orbits), $F_{i}$ and $G_{j}$, are distinct and are both $A$-canonical, then $F_{i}$ and $G_{j}$ are nonisomorphic. Also, if $F_{i}=\left(O_{1}, O_{2}, \ldots, O_{i}\right)$ is $A$-canonical, and $1 \leq j \leq i$, then $F_{j}=\left(O_{1}, O_{2}, \ldots, O_{j}\right)$ is also $A$-canonical.

Let $N(A)$ be the normalizer group of $A$ within $\mathbf{S}_{2 n}$; that is, $N(A)=\left\{\pi: \pi^{-1} A \pi\right.$ $\left.=A, \pi \in \mathbf{S}_{2 n}\right\}$. It is easy to see that $\pi \in N(A)$ maps any eligible orbit into an eligible orbit of the same length. It should be noted that for a given $F_{i}$ we have that $N(A) \leq M\left(F_{i}\right)$, and in general $|N(A)| \ll\left|M\left(F_{i}\right)\right|$.

We say $F_{i}$ is quasi-A-canonical if $F_{i}^{\alpha} \geq F_{i}$ for all $\alpha \in N(A)$. A quasi- $A$-canonical $F_{i}$ may not be $A$-canonical, since it is possible to have the situation where all the mappings that take $F_{i}$ into an isomorphic copy are not in $N(A)$ (for example, refer to case 20 of Table 3 ).

3. Orderly Algorithms. In this section we outline the orderly algorithms that were used to obtain the results of this paper. These algorithms are modifications of the algorithms described in [13] and [14].

There are two ways to generate the OFs: the breadth-first and the depth-first algorithms, as described in the following paragraphs (see also [13] and [14]). We use $N(A)$ instead of $M\left(F_{i}\right)$ (for a given $F_{i}$ ) to eliminate isomorphic structures, for the following two reasons: (1) the number of mappings to be performed is significantly reduced; (2) recalculation of $M\left(F_{i}\right)$ is avoided when $F_{i}$ changes. However, the OFs thus generated are not necessarily nonisomorphic. An additional step is therefore required to identify and eliminate the isomorphic copies of these OFs: We first find the canonical representation of these OFs, and then delete any duplications.

(a) Breadth-first algorithm: The following pseudo-code describes how to generate $\mathbf{F}_{i+1}$ from $\mathbf{F}_{i}$, where $\mathbf{F}_{i}$ is the set of all proper partial OFs containing $i$ orbits that are quasi- $A$-canonical. Note that $\mathbf{F}_{0}=\{\varnothing\}$.

$\mathbf{F}_{i+1}=\varnothing$

FOR each $F_{i} \in \mathbf{F}_{i}$ DO 
Determine the smallest integer $j$ such that the edge $(1, j+1)$ is not in $F_{i}$;

FOR each orbit $O$ whose representative is in $\mathbf{U}_{j} \mathrm{DO}$

IF the one-factors of $O$ are disjoint from the one-factors in $F_{i}$ THEN

FOR each $\pi \in N(A)$ DO

(1) compute $O^{\pi}$ and $F_{i}^{\pi}$

(2) IF $F_{i}^{\pi} \cup\left\{O^{\pi}\right\}<F_{i} \cup\{O\}$ THEN

$F_{i} \cup\{O\}$ is not canonical, discard it and go on to next $O$;

$\left\{\right.$ Here $F_{i}^{\pi} \cup\left\{O^{\pi}\right\} \geq F_{i} \cup\{O\}$ for all $\pi$, save $F_{i} \cup\{O\}$ for the next step. $\}$

$$
\mathbf{F}_{i+1}=\mathbf{F}_{i+1} \cup\left\{F_{u} \cup\{O\}\right\} \text {. }
$$

(b) Depth-first algorithm: The following recursive pseudo-code outlines how to generate from a given $F_{i}$ all quasi- $A$-canonical OFs extending $F_{i}$ :

PROCEDURE Depth-first $\left(F_{i}\right)$

Determine the smallest integer $j$ such that the edge $(1, j+1)$ is not in $F_{i}$; IF $j=2 n+1$ THEN

$F_{i}$ is a quasi- $A$-canonical $\mathrm{OF}$;

ELSE

FOR each orbit $O$ whose representative is in $\mathbf{U}_{j} \mathrm{DO}$

IF the one-factors of $O$ are disjoint from the one-factors of $F_{i}$ THEN IF $F_{i}^{\pi} \cup\left\{O^{\pi}\right\} \geq F_{i} \cup\{O\}$ for all $\pi \in N(A)$ THEN

Depth-first $\left(F_{i} \cup\{O\}\right)$.

We remark that when $A$ is the trivial group of order one, all one-factors are eligible orbits of length one. Consequently, these two algorithms reduce to the orderly algorithms described in [13] and [14]. In this case, we would obtain the complete enumeration of the OFs of $K_{2 n}$.

Note that the algorithms above can be easily modified for subclasses of OFs that may be of interest. For example, to enumerate perfect OFs, we modify the algorithms so that pairs of distinct one-factors are both disjoint and Hamiltonian.

4. One-Factorizations of $K_{12}$. Let $a$ be a permutation of $\{1, \ldots, 12\}$ and define $A=\langle a\rangle$. The generator $a$ of the cyclic group $A$ on 12 elements can have one of 77 different cycle structures (refer to Table 2). Many of these cases can be eliminated easily by the following general results on the cycle structure of automorphisms of OFs of $K_{2 n}$.

LEMMA 4.1. If $a$ is an automorphism of an $O F$ of $K_{2 n}$, then the number of fixed points in a is even or equals 1.

Proof. Suppose the number of fixed points of $a$ is $2 k+1(k \geq 1)$, and let the fixed points be $p_{1}, p_{2}, p_{3}, \ldots, p_{2 k+1}$. Consider the one-factor $f$ containing the edge $\left\{p_{1}, p_{2}\right\}$. Then $f$ must be an orbit of length one. But then there exists an edge $\left\{p_{i}, q_{j}\right\}$ ( $q_{j}$ is not a fixed point) in $f$ which maps into an edge of another one-factor; hence a contradiction. 
LEMMA 4.2. If $a$ is an automorphism of $K_{2 n}$ and has more than $n$ fixed points, then the number of fixed points in a is $2 n$.

Proof. Let the number of fixed points be $2 k$, where $2 k>n$. Then there exists an edge of two fixed points in every one-factor of the OF. Every one-factor is thus an orbit of length one. Consequently, each one-factor has $k$ edges made up of the $2 k$ fixed points and it is impossible to have an edge of the form $\left\{p_{i}, q_{j}\right\}$, where $p_{i}$ is a fixed point and $q_{j}$ is not a fixed point (except the case when all the points in $a$ are fixed points).

LEMMA 4.3. If $a$ is an automorphism of an $O F$ of $K_{2 n}$ and has exactly $n$ fixed points, then the remaining $n$ points of a must appear as disjoint 2-cycles.

Proof. Consider the one-factors that are fixed by $a$. Each of these one-factors has $n / 2$ edges made up of fixed points, so there are exactly $n-1$ such one-factors.

The remaining $n$ one-factors consist of edges of the form $\left\{p_{i}, q_{j}\right\}$, where $p_{i}$ is a fixed point and $q_{j}$ is not a fixed point. Therefore, all edges made up of nonfixed points, $\left\{q_{i}, q_{j}\right\}$, must appear in the $n-1$ fixed one-factors. Consequently, the nonfixed points can only appear as disjoint 2-cycles in $a$.

COROLLARY. If $n \equiv 2(\bmod 4), n>2$, then the number of fixed points in a is not equal to $n$.

Proof. Consider the $n-1$ one-factors that are fixed by $a$. Each of these onefactors has $n / 2$ edges from the $n$ points in the 2 -cycles. Since $n \equiv 2(\bmod 4)$, each of these one-factors must have at least one edge of the form $\left\{c_{1}, c_{2}\right\}$, where $c_{1}$ and $c_{2}$ appear in the same 2-cycle. Now there are $n / 22$-cycles (edges) to be filled in these $n-1$ one-factors. So $(n / 2) \geq(n-1)$, or $n \leq 2$.

LEMMA 4.4. If $a$ is a nonidentity automorphism of an $O F$ of $K_{2 n}$ and has no fixed points, then the number of 3-cycles in a does not equal 1.

Proof. Consider a 3-cycle $(b c d)$. Edges $\{b, c\},\{c, d\}$, and $\{d, b\}$ appear in 3 distinct one-factors forming an orbit of length 3 . Thus we have

$$
\begin{aligned}
& \{b, c\} \rightarrow\{c, d\} \rightarrow\{d, b\} \rightarrow\{b, c\} \\
& \{d, x\} \rightarrow\{b, y\} \rightarrow\{c, z\} \rightarrow\{d, x\}
\end{aligned}
$$

and $(x y z)$ is another 3 -cycle.

LEMMA 4.5. Let $a$ be an automorphism of an OF of $K_{2 n}$. If the number of fixed points in $a$ is even and nonzero, and the remaining points form a cycle, then there must be exactly two fixed points in a.

Proof. Let the number of fixed points be $2 k$, then the number of nonfixed points is $2 n-2 k$ and they form a cycle $\left(q_{1} q_{2} q_{3} \cdots q_{2 n-2 k}\right)$. Consider the onefactors containing the edge $\left\{p_{i}, p_{j}\right\}$ made up of fixed points: There are $2 k-1$ of these one-factors. There is only one way that the nonfixed points may appear in these $2 k-1$ one-factors: They must appear as edges $\left\{q_{1}, q_{n-k+1}\right\},\left\{q_{2}, q_{n-k+2}\right\}$, $\left\{q_{3}, q_{n-k+3}\right\}, \ldots$, and $\left\{q_{n-k}, q_{2 n-2 k}\right\}$. Thus $2 k-1=1$ and hence the number of fixed points is two. 
LEMMA 4.6. Let a be an automorphism of an $O F$ of $K_{2 n}$. If there is a 2-cycle (bc) in a, then the OF has an orbit of length one.

Proof. The one-factor containing the edge $\{b, c\}$ must be fixed by $a$.

COROLLARY. If a has exactly one fixed point, then there cannot be an 2-cycles in $a$.

Proof. If $a$ has exactly one fixed point, then there does not exist any one-factor fixed by $a$. Consequently, there cannot be any 2-cycles in $a$.

LEMMA 4.7. Let $a$ be an automorphism of an OF of $K_{2 n}$. If a has two cycles of lengths $L 1$ and $L 2(L 1<L 2)$, then $\operatorname{LCM}(L 1, L 2) \leq 2 n-1$.

Proof. Let the $L 1$-cycle be denoted $\left(p_{1} p_{2} \ldots p_{L 1}\right)$, and the $L 2$-cycle be denoted by $\left(q_{1} q_{2} \ldots q_{L 2}\right)$. Since $L 1 \neq L 2$, the one-factor $f$ containing the edge $\left\{p_{1}, q_{1}\right\}$ is in an orbit of length greater than one. So $f$ maps into another one-factor containing the edge $\left\{p_{2}, q_{2}\right\}$, which in turn maps into the one-factor containing the edge $\left\{p_{3}, q_{3}\right\}$, and so on. Thus the one-factor containing $\left\{p_{1}, q_{1}\right\}$ is in an orbit of length $\operatorname{LCM}(L 1, L 2)$. Hence $\operatorname{LCM}(L 1, L 2) \leq 2 n-1$.

THEOREM 4.8. There are at most 18 cycle structures of a that admit OFs for $K_{12}$.

Proof. Using Lemmas 4.1 to 4.7, we eliminated all but 29 cases (refer to Table 2 ). Of these 29 cases, we can eliminate 11 further cases, by observing that $a^{n}$ for some $n>1$ is not an admissible automorphism. As an example, for case 24, $a$ has cycle structure $6^{1} 3^{2}$. But then $a^{3}$ has cycle structure $2^{3} 1^{6}$, which is case 74 and is ruled out by the corollary to Lemma 4.3 .

For those cases that are not eliminated by the above lemmas and hence may admit OFs, we resort to the help of computer. We implemented the two algorithms in PASCAL/VS on the Amdahl/580 computer at the University of Manitoba. All the cases in Table 2 except cases 71 and 77 are dealt with in this paper. For cases 72 and 73 , we first used the breadth-first algorithm to construct $\mathbf{F}_{4}$, then extended the proper partial OFs in $\mathbf{F}_{4}$ to complete OFs by the depth-first algorithm. For the other cases, only the depth-first algorithm was used.

Both cases 71 and 77 require a large amount of computing time. Case 77 is equivalent to a complete enumeration of the OFs of $K_{12}$, which is out of our reach at this point in time. Case 71 involves constructing OFs containing automorphisms of six 2-cycles. Instead of dealing with case 71 in its entirety, we looked at the subproblem of the enumeration of OFs that contain two automorphisms of six 2cycles. That is, we have $A=\left\langle a_{1}, a_{2}\right\rangle$, where $a_{1}=((12)(34)(56)(78)(910)(1112))$ and $\left.a_{2}=(13)(24)(57)(68)(911)(1012)\right)$. (It turns out that, up to isomorphism, this is the only admissible case for given $a_{1}$.) We refer to this as case 78 in Table 3. Similar to cases 72 and 73 , we used the combination of breadth-first and depth-first algorithms.

Therefore, we have enumerated all OFs of $K_{12}$ except those containing exactly one automorphism of six 2-cycles and those with the trivial automorphism group.

In Table 3 we list the cases that admit at least one OF and the associated statistics. It is interesting to note that there are 6 cases where $N(A)$ did not eliminate all the isomorphic OFs (cases 20, 44, 46, 47, 73 and 78). 
Interested in finding out what mappings would have eliminated these isomorphic OFs, we looked into case 20, where 30 pairs of isomorphic OFs survived the test of $N(A)$. Here, $A=\langle(123456)(789101112)\rangle$.

Of these 30 pairs of OFs, 6 of them have the full automorphism groups of order 12 , and 21 have order 24 . The automorphism groups of these 27 pairs of OFs each contain a unique cyclic subgroup $B=\langle(135)(246)(7911)((81012)\rangle$. Since $B$ is unique, any $\alpha$ that takes an OF into its isomorphic copy must also map $B$ into $B$; that is, $\alpha \in N(B)$. Thus, if we use $N(B)$ instead of $N(A)$, we would be able to eliminate the 27 isomorphic OFs.

Each of the remaining 3 pairs of OFs has a full automorphism group of order 48 , and each has 4 copies of $\mathbf{Z}_{3}$ in its automorphism group. In each of these three cases, there exists an $\alpha \in N(B)$ which takes an OF into is isomorphic copy. Here again, using $N(B)$ would have eliminated the 3 isomorphic OFs.

It should be emphasized that, in general, we do not know what the full automorphism groups look like beforehand. Consequently, the best strategy is perhaps to use the normalizer of the prescribed subgroup $N(A)$ to obtain the quasi- $A$-canonical OFs, followed by testing these OFs for isomorphism. The statistics on $K_{12}$ indicates that $N(A)$ is able to get rid of most of the isomorphic OFs. We would like to point out that, in certain situations, however, the normalizer of the prescribed group $N(A)$ is sufficient to eliminate isomorphic OFs; that is, quasi- $A$-canonical OFs are nonisomorphic in these cases. This result is as follows (see [8] and [11]):

THEOREM 4.9. Suppose two OFs $F$ an $G$ of $K_{n+1}$ contain $\mathbf{Z}_{n}$ in their automorphism groups, where $n$ is an odd prime or the product of two distinct odd primes. If $F$ is isomorphic to $G$, then $F^{\alpha}=G$ for some $\alpha \in N\left(\mathbf{Z}_{n}\right)$.

Thus for $A=\mathbf{Z}_{11}$, the OFs of $K_{12}$ constructed with the use of $N(A)$ are nonisomorphic (case 2 in Tables 2 and 3 ).

TABLE 1

Frequency distribution of the orders of automorphism groups of OFs of $K_{12}$

\begin{tabular}{rr}
\hline Order & No. \\
2 & $\geq 39706$ \\
3 & 669 \\
4 & 14801 \\
5 & 92 \\
6 & 245 \\
8 & 610 \\
10 & 10 \\
11 & 2 \\
12 & 138 \\
16 & 76 \\
20 & 2 \\
24 & 25 \\
32 & 4 \\
48 & 6 \\
55 & 1 \\
110 & 1 \\
240 & 2 \\
660 & 1 \\
\hline & 56391
\end{tabular}


Table 1 gives the distribution of the orders of automorphism groups for the OFs of $K_{12}$ constructed in this $\mathrm{p}^{\circ}$,eer. Note that the numbers in Table 1 are exact, with the exception of the number of OFs of order 2 .

The CPU time for all cases dealt with in this paper, except 72, 73 and 78, added up to about 40 minutes. Case 72 took 7.5 hours, case 73 needed 30 hours, and case 78 consumed about 17 hours. These timings include the final step to determine the canonical representations and eliminate the isomorphic OFs.

5. Perfect One-Factorizations of $K_{14}$. The algorithms outlined in Section 2 were also used to construct perfect OFs of $K_{14}$, and some results were reported in [14]. Here, we give a complete report of our findings.

We were able to prove that there are exactly 21 perfect OFs of $K_{14}$ with nontrivial automorphism groups. In fact, the algorithms described in this paper helped find 6 new perfect OFs (sets 16-20 in [14], and set 21 in Table 5), in addition to the 11 new perfect OFs found by the orderly algorithms in [14].

We started by looking at the cycle structure of the generator $a$ of the cyclic group on 14 elements. In total, there are 135 possibilities. Many of them can be eliminated easily. In fact, Ihrig proved in [6] that the cycle structure of $a$ must be one of the forms given in the following lemma.

LEMMA 5.1. If $a$ is an automorphism of a perfect $O F$ of $K_{2 n}$, then its cycle structure must be one of the four forms: (1) $1^{2} k^{(2 n-2) / k}$, (2) $1^{1} k^{(2 n-1) / k}$, (3) $k^{2 n / k}$, or (4) $2^{1} k^{(2 n-2) / k}$.

Proof. See [6, Corollary 3.4].

THEOREM 5.2. There are at most 13 cycle structures of a that admit perfect OFs for $K_{14}$.

Proof. By Lemma 5.1, there are at most 14 cycle structures of $a$ that admit perfect OFs for $K_{14}$. These are:
(1) $14^{1}$
(2) $13^{1} 1^{1}$
(3) $12^{1} 2^{1}$
(4) $12^{1} 1^{2}$
(5) $7^{2}$
(6) $6^{2} 2^{1}$
(7) $6^{2} 1^{2}$
(8) $4^{3} 2^{1}$
(9) $4^{3} 1^{2}$
(10) $3^{4} 2^{1}$
(11) $3^{4} 1^{2}$
(12) $2^{7}$
(13) $2^{6} 1^{2}$
(14) $1^{14}$.

Case (10) can be eliminated since $a^{3}$ has the form $2^{1} 1^{12}$, which is not admissible.

We list in Table 4 those cases that admit at least one perfect OF for $K_{14}$ and the associated statistics. As in $K_{12}$, we omit the case involving the trivial automorphism (case 14). In total, approximately 10 hours of computer time was required for the remaining 12 cases (including the time required to determine the canonical representation of the perfect OFs constructed).

Of special interest is case 12 (where $a=((12)(34) \cdots(1314))$ ). It is not difficult to see that the seven edges from the seven 2-cycles of $a$ must either (i) appear in the same one-factor, or (ii) appear in seven distinct one-factors. An OF of type (i) would have one orbit of one-factors of length 1 and six orbits of length 2 , and type (ii) would have seven orbits of length 1 and three orbits of length 2 . In using orderly algorithms for these two subcases, we omit canonicity testing $(645120$ mappings would have been needed for each $F_{i}$ ), and test the OFs for isomorphism after they have been created. 
TABLE 2

Cycle structures of admissible automorphisms of OFs of $K_{12}$

\begin{tabular}{|c|c|c|c|c|c|}
\hline $\begin{array}{l}\text { Case } \\
\text { no. }\end{array}$ & $\begin{array}{l}\text { Cycle } \\
\text { structure }\end{array}$ & $\begin{array}{l}\text { Eliminated by } \\
\text { lemma }\end{array}$ & $\begin{array}{l}\text { Case } \\
\text { no. }\end{array}$ & $\begin{array}{l}\text { Cycle } \\
\text { structure }\end{array}$ & $\begin{array}{l}\text { Eliminated by } \\
\text { lemma }\end{array}$ \\
\hline 1 & $12^{1}$ & & 2 & $11^{1} 1^{1}$ & \\
\hline 3 & $10^{1} 2^{1}$ & & 4 & $10^{1} 1^{2}$ & \\
\hline 5 & $9^{1} 3^{1}$ & 4.4 & 6 & $9^{1} 2^{1} 1^{1}$ & 4.7 \\
\hline 7 & $9^{1} 1^{3}$ & 4.1 & 8 & $8^{1} 4^{1}$ & \\
\hline 9 & $8^{1} 3^{1} 1^{1}$ & 4.7 & 10 & $8^{1} 2^{2}$ & \\
\hline 11 & $8^{1} 2^{1} 1^{2}$ & & 12 & $8^{1} 1^{4}$ & 4.5 \\
\hline 13 & $7^{1} 5^{1}$ & 4.7 & 14 & $7^{1} 4^{1} 1^{1}$ & 4.7 \\
\hline 15 & $7^{1} 3^{1} 2^{1}$ & 4.4 & 16 & $7^{1} 3^{1} 1^{2}$ & 4.7 \\
\hline 17 & $7^{1} 2^{2} 1^{1}$ & 4.6 & 18 & $7^{1} 2^{1} 1^{3}$ & 4.1 \\
\hline 19 & $7^{1} 1^{5}$ & 4.1 & 20 & $6^{2}$ & \\
\hline 21 & $6^{1} 5^{1} 1^{1}$ & 4.7 & 22 & $6^{1} 4^{1} 2^{1}$ & 4.7 \\
\hline 23 & $6^{1} 4^{1} 1^{2}$ & 4.7 & 24 & $6^{1} 3^{2}$ & $a^{3}($ case 74$)$ \\
\hline 25 & $6^{1} 3^{1} 2^{1} 1^{1}$ & 4.6 & 26 & $6^{1} 3^{1} 1^{3}$ & 4.1 \\
\hline 27 & $6^{1} 2^{3}$ & $a^{2}($ case 65$)$ & 28 & $6^{1} 2^{2} 1^{2}$ & $a^{2}($ case 65$)$ \\
\hline 29 & $6^{1} 2^{1} 1^{4}$ & $a^{2}$ (case 65$)$ & 30 & $6^{1} 1^{6}$ & 4.3 \\
\hline 31 & $5^{2} 2^{1}$ & $a^{5}($ case 76$)$ & 32 & $5^{2} 1^{2}$ & \\
\hline 33 & $5^{1} 4^{1} 3^{1}$ & 4.4 & 34 & $5^{1} 4^{1} 2^{1} 1^{1}$ & 4.6 \\
\hline 35 & $5^{1} 4^{1} 1^{3}$ & 4.1 & 36 & $5^{1} 3^{2} 1^{1}$ & 4.7 \\
\hline 37 & $5^{1} 3^{1} 2^{2}$ & 4.4 & 38 & $5^{1} 3^{1} 2^{1} 1^{2}$ & 4.7 \\
\hline 39 & $5^{1} 3^{1} 1^{4}$ & 4.7 & 40 & $5^{1} 2^{3} 1^{1}$ & 4.6 \\
\hline 41 & $5^{1} 2^{2} 1^{3}$ & 4.1 & 42 & $5^{1} 2^{1} 1^{5}$ & 4.1 \\
\hline 43 & $5^{1} 1^{7}$ & 4.1 & 44 & $4^{3}$ & \\
\hline 45 & $4^{2} 3^{1} 1^{1}$ & 4.7 & 46 & $4^{2} 2^{2}$ & \\
\hline 47 & $4^{2} 2^{1} 1^{2}$ & & 48 & $4^{2} 1^{4}$ & \\
\hline 49 & $4^{1} 3^{2} 2^{1}$ & 4.7 & 50 & $4^{1} 3^{2} 1^{2}$ & 4.7 \\
\hline 51 & $4^{1} 3^{1} 2^{2} 1^{1}$ & 4.6 & 52 & $4^{1} 3^{1} 2^{1} 1^{3}$ & 4.1 \\
\hline 53 & $4^{1} 3^{1} 1^{5}$ & 4.1 & 54 & $4^{1} 2^{4}$ & $a^{2}($ case 75$)$ \\
\hline 55 & $4^{1} 2^{3} 1^{2}$ & $a^{2}($ case 75$)$ & 56 & $4^{1} 2^{2} 1^{4}$ & $a^{2}($ case 75$)$ \\
\hline 57 & $4^{1} 2^{1} 1^{6}$ & 4.3 & 58 & $4^{1} 1^{8}$ & 4.2 \\
\hline 59 & $3^{4}$ & & 60 & $3^{3} 2^{1} 1^{1}$ & 4.6 \\
\hline 61 & $3^{3} 1^{3}$ & 4.1 & 62 & $3^{2} 2^{3}$ & $a^{3}($ case 74$)$ \\
\hline 63 & $3^{2} 2^{2} 1^{2}$ & $a^{3}($ case 75$)$ & 64 & $3^{2} 2^{1} 1^{4}$ & $a^{3}($ case 76$)$ \\
\hline 65 & $3^{2} 1^{6}$ & 4.3 & 66 & $3^{1} 2^{4} 1^{1}$ & 4.6 \\
\hline 67 & $3^{1} 2^{3} 1^{3}$ & 4.1 & 68 & $3^{1} 2^{2} 1^{5}$ & 4.1 \\
\hline 69 & $3^{1} 2^{1} 1^{7}$ & 4.1 & 70 & $3^{1} 1^{9}$ & 4.1 \\
\hline 71 & $2^{6}$ & & 72 & $2^{5} 1^{2}$ & \\
\hline 73 & $2^{4} 1^{4}$ & & 74 & $2^{3} 1^{6}$ & 4.3 \\
\hline 75 & $2^{2} 1^{8}$ & 4.2 & 76 & $2^{1} 1^{10}$ & 4.2 \\
\hline 77 & $1^{12}$ & & & & \\
\hline
\end{tabular}

In [6], Ihrig defined a $P$ element to be an automorphism of $n$ 2-cycles of a perfect OF of $K_{2 n}$, the cycles of which form the edges of a one-factor of the perfect OF. Thus, an OF of type (i) contains a $P$ element. Ihrig observed that, other than the perfect $\mathrm{OF}$ of $K_{4}$, there is no other example known of a perfect OF of $K_{2 n}$ containing a $P$ element. Our computer search did not find any perfect $\mathrm{OF}$ of type (i) for $K_{14}$, suggesting that perhaps perfect OFs of $K_{2 n}$ containing a $P$ element do not exist for $n>2$. 
There are 165 perfect OFs of type (ii), of which 4 are nonisomorphic. The information on the number of orbits and distinct one-factors listed in Table 4 for case 12 pertains to type (ii).

It is interesting to note that, except for case 12 , the quasi- $A$-canonical perfect OFs constructed from each of the other cases turn out to be nonisomorphic (that is, they are also $A$-canonical).

We list in Table 5 the perfect OF of $K_{14}$ (set 21) that was not reported in [14]. In [6] and [7], Ihrig studied the order of full automorphism groups of perfect OFs of $K_{2 n}$ (see also [14]). The automorphism groups of the 21 perfect OFs of $K_{14}$ (see [14] and Table 5) give examples of every possible group order permissible by the results of Ihrig (except order 1).

TABLE 3

One-factorizations of $K_{12}$ containing prescribed automorphism groups

\begin{tabular}{|c|c|c|c|c|c|c|c|c|c|}
\hline \multirow[b]{2}{*}{$\begin{array}{l}\text { Case } \\
\text { no. }\end{array}$} & \multirow[b]{2}{*}{$\begin{array}{l}\text { Cycle } \\
\text { struc- } \\
\text { ture } \\
\text { of } a\end{array}$} & \multirow[b]{2}{*}{$|N(A)|$} & \multirow[b]{2}{*}{$\begin{array}{l}\text { No. of } \\
\text { distinct } \\
\text { orbits }\end{array}$} & \multirow[b]{2}{*}{$\begin{array}{l}\text { No. of } \\
\text { distinct } \\
\text { 1-factors }\end{array}$} & \multicolumn{3}{|c|}{ Quasi-A-canonical OF } & \multicolumn{2}{|c|}{$A$-canonical OF } \\
\hline & & & & & Total & $\begin{array}{l}\text { Not in } \\
\text { prev. } \\
\text { cases } \\
(2)\end{array}$ & $\begin{array}{l}\text { In } \\
\text { prev. } \\
\text { cases } \\
(3)\end{array}$ & $\begin{array}{l}\text { Not in } \\
\text { prev. } \\
\text { cases } \\
(4)\end{array}$ & $\begin{array}{l}\text { In } \\
\text { prev. } \\
\text { cases } \\
(5)\end{array}$ \\
\hline
\end{tabular}

\begin{tabular}{llrrrrrrrr}
\hline 1 & $12^{1}$ & 48 & 19 & 79 & 6 & 6 & 0 & 6 & 0 \\
2 & $11^{1} 1^{1}$ & 110 & 25 & 275 & 5 & 5 & 0 & 5 & 0 \\
3 & $10^{1} 2^{1}$ & 80 & 17 & 81 & 3 & 2 & 1 & 2 & 1 \\
4 & $10^{1} 1^{2}$ & 80 & 57 & 561 & 7 & 6 & 1 & 6 & 1 \\
11 & $8^{1} 2^{1} 1^{2}$ & 128 & 133 & 1033 & 12 & 12 & 0 & 12 & 0 \\
20 & $6^{2}$ & 144 & 221 & 1073 & 297 & 287 & 10 & 227 & 8 \\
32 & $5^{2} 1^{2}$ & 400 & 905 & 4505 & 109 & 97 & 12 & 97 & 12 \\
44 & $4^{3}$ & 768 & 709 & 2557 & 390 & 381 & 9 & 376 & 8 \\
46 & $4^{2} 2^{2}$ & 512 & 399 & 1551 & 76 & 74 & 2 & 64 & 2 \\
47 & $4^{2} 2^{1} 1^{2}$ & 256 & 565 & 2213 & 328 & 291 & 37 & 273 & 31 \\
48 & $4^{2} 1^{4}$ & 1536 & 783 & 3087 & 222 & 173 & 49 & 173 & 49 \\
59 & $3^{4}$ & 3888 & 1953 & 5805 & 1086 & 850 & 236 & 850 & 236 \\
72 & $2^{5} 1^{2}$ & 7680 & 2561 & 5041 & 5676 & 5665 & 11 & 5665 & 11 \\
73 & $2^{4} 1^{4}$ & 9216 & 1803 & 3531 & 38751 & 38029 & 722 & 37063 & 598 \\
78 & $2^{6} \times 2^{6}$ & 2304 & 399 & 927 & 13341 & 11572 & 1769 & 11572 & 695 \\
& & & & & & & & & 56391
\end{tabular}

(1) $\quad=(2)+(3)$.

(2)-(4) gives the number of isomorphic OFs (not appearing in previous cases) which are not eliminated by $N(A)$.

(3)-(5) gives the number of isomorphic OFs (appearing in previous cases) which are not eliminated by $N(A)$. 
TABLE 4

Perfect one-factorizations of $K_{14}$ containing prescribed automorphism groups

\begin{tabular}{|c|c|c|c|c|c|c|}
\hline $\begin{array}{l}\text { Case } \\
\text { no. }\end{array}$ & $\begin{array}{l}\text { Cycle } \\
\text { structure } \\
\text { of } a \\
\end{array}$ & $|N(A)|$ & $\begin{array}{l}\text { No. of } \\
\text { distinct } \\
\text { orbits } \\
\end{array}$ & $\begin{array}{l}\text { No. of } \\
\text { distinct } \\
\text { 1-factors }\end{array}$ & $\begin{array}{l}--- \\
\text { total } \\
\text { gene- } \\
\text { rated }\end{array}$ & $\begin{array}{l}\text { Nonisomorphic OF }--- \\
\text { set no. in }[14]\end{array}$ \\
\hline 1 & $14^{1}$ & 84 & 12 & 63 & 1 & set 1 \\
\hline 2 & $13^{1} 1^{1}$ & 156 & 1 & 13 & 1 & set 13 \\
\hline 4 & $12^{1} 1^{2}$ & 96 & 25 & 289 & 3 & sets $13,14,15$ \\
\hline 5 & $7^{2}$ & 294 & 565 & 3913 & 1 & set 1 \\
\hline 7 & $6^{2} 1^{2}$ & 288 & 1399 & 8359 & 12 & sets $1,3-6,9-15$ \\
\hline 9 & $4^{3} 1^{2}$ & 1536 & 4621 & 18445 & 5 & sets $9,10,13,14,15$ \\
\hline 11 & $3^{4} 1^{2}$ & 7776 & 15579 & 46683 & 17 & sets $1,3-6,9-20$ \\
\hline 12 & $2^{7}$ & 645120 & 23880 & 46920 & 4 & sets $1,2,3,21$ (Table 5 ) \\
\hline 13 & $2^{6} 1^{2}$ & 92160 & 32395 & 64659 & 15 & sets $1-15$ \\
\hline
\end{tabular}

TABLE 5

Perfect one-factorization of $K_{14}$ (set 21)

$|A|=2$.

$A=\langle a\rangle$;

$a=(14)(25)(37)(610)(812)(913)(1114)$

$a$ induces $\left(f_{2} f_{7}\right)\left(f_{6} f_{12}\right)\left(f_{9} f_{14}\right)$

$\begin{array}{rrrrrrrrrrrrrr}1 & 2 & 3 & 4 & 5 & 6 & 7 & 8 & 9 & 10 & 11 & 12 & 13 & 14 \\ 1 & 3 & 2 & 5 & 4 & 7 & 6 & 9 & 8 & 11 & 10 & 13 & 12 & 14 \\ 1 & 4 & 2 & 6 & 3 & 8 & 5 & 10 & 7 & 12 & 9 & 14 & 11 & 13 \\ 1 & 5 & 2 & 4 & 3 & 9 & 6 & 11 & 7 & 13 & 8 & 12 & 10 & 14 \\ 1 & 6 & 2 & 14 & 3 & 5 & 4 & 8 & 7 & 9 & 10 & 11 & 12 & 13 \\ 1 & 7 & 2 & 10 & 3 & 12 & 4 & 5 & 6 & 13 & 8 & 14 & 9 & 11 \\ 1 & 8 & 2 & 11 & 3 & 6 & 4 & 12 & 5 & 14 & 7 & 10 & 9 & 13 \\ 1 & 9 & 2 & 13 & 3 & 10 & 4 & 11 & 5 & 12 & 6 & 8 & 7 & 14 \\ 1 & 10 & 2 & 3 & 4 & 6 & 5 & 7 & 8 & 13 & 9 & 12 & 11 & 14 \\ 1 & 11 & 2 & 9 & 3 & 7 & 4 & 14 & 5 & 13 & 6 & 12 & 8 & 10 \\ 1 & 12 & 2 & 7 & 3 & 13 & 4 & 10 & 5 & 11 & 6 & 14 & 8 & 9 \\ 1 & 13 & 2 & 12 & 3 & 14 & 4 & 9 & 5 & 8 & 6 & 10 & 7 & 11 \\ 1 & 14 & 2 & 8 & 3 & 11 & 4 & 13 & 5 & 9 & 6 & 7 & 10 & 12\end{array}$

Department of Computer Science

University of Manitoba

Winnipeg, Manitoba R3T 2N2, Canada

1. P. J. CAmeron, "Minimal edge-colourings of complete grahs," J. London Math. Soc. (2), v. 11, 1975, pp. 337-346.

2. P. J. Cameron, Parallelisms of Complete Designs, London Math. Soc. Lecture Note Ser., vol. 23, Cambridge Univ. Press, Cambridge, 1976.

3. L. E. DiCKSON \& F. H. SAFFORD, "Solution to problem 8 (group theory)," Amer Math. Monthly, v. 13, 1906, pp. 150-151.

4. E. N. Gelling, On 1-factorizations of the Complete Graph and the Relationship to Round Robin Schedules, M.A. Thesis, University of Victoria, 1973.

5. F. HARARY, Graph Theory, Addison-Wesley, Reading, Mass., 1969.

6. E. IHRIG, "Symmetry groups related to the construction of perfect one-factorizations of $K_{2 n}$," J. Combin. Theory Ser. B, v.40, 1986, pp. 121-151.

7. E. IHRIG, "The structure of the symmetry groups of perfect one-factorizations of $K_{2 n}$." (To appear.) 
8. W. L. Kocay, D. R. Stinson \& S. A. VAnstone, "On strong starters in cyclic groups," Discrete Math., v. 56, 1985 , pp. 45-60.

9. C. C. LindNER, E. MENDELSOHN \& A. ROSA, "On the number of 1-factorizations of the complete graph," J. Combin. Theory, v. 20, 1976, pp. 265-282.

10. E. MENDELSOHN \& A. ROSA, "One-factorizations of the complete graph-A survey," $J$. Graph Theory, v. 9, 1985, pp. 43-65.

11. K. T. Phelps \& S. A. VAnstone, "Isomorphism of strong starters in cyclic groups." (Preprint.)

12. R. C. READ, "Every one a winner," Ann. Discrete Math., v. 2, 1978, pp. 107-120.

13. E. SEAH \& D. R. STINSON, "An enumeration of non-isomorphic one-factorizations and Howell designs for the graph $K_{10}$ minus a one-factor," Ars Combin., v. 21, 1986, pp. 145-161.

14. E. SEAH \& D. R. Stinson, "Some perfect one-factorizations of $K_{14}$," Ann. Discrete Math. (To appear.)

15. W. D. WAllis, A. P. Street \& J. S. WAllis, Combinatorics: Room Squares, Sum-Free Sets, Hadamard Matrices, Lecture Notes in Math., vol. 292, Springer-Verlag, New York, 1972.

16. W. D. WAllis, "On one-factorizations of complete graphs," J. Austral. Math. Soc., v. 16, 1973, pp. 167-171. 